\title{
Sex Differences in the Daily Pattern of Locomotor Activity in Laboratory Rats
}

\author{
F. Wollnik \\ Institut für Versuchstierkunde im Zentralen Tierlaboratorium \\ der Medizinischen Hochschule, D-3000 Hannover
}

Recent studies have shown that steroid hormones modulate circadian timekeeping processes in mammals [1]. Furthermore, the neuronal structure of the suprachiasmatic nuclei of the hypothalamus, which are essential for the generation and entrainment of circadian rhythms, appears to be sexually differentiated [2]. Although this morphological dimorphism is suspected to cause sex differences in the circadian control of various functions, only few studies have addressed this question [3-6].

The present study supplies information supporting an obvious sex difference in the daily pattern of locomotor activity in laboratory rats. Ultradian rhythms, which have been found to be genetically fixed in two inbred strains of rats [7], are also sex-specific. They were observed in male animals of these strains. The females failed to show any ultradian rhythmicity.
Adult male and female rats of the inbred strains $\mathrm{BH} / \mathrm{Ztm}(\hat{\sigma} / \%, n=6)$ and LEW/Ztm (ô, $n=12$;,$+ n=11)$ were bred in our laboratory on a light-dark cycle of LD 12:12 under controlled environmental conditions (room temperature $22 \pm 1{ }^{\circ} \mathrm{C}$, relative humidity $55 \pm 5 \%$ ). Locomotor activity was measured continuously for 10 days with an electronic movement analyzer [8]. The animals were individually kept in polycarbonate cages $(55 \times 33 \times 20 \mathrm{~cm})$ with free access to food and water. The periodicity of the activity patterns was examined using the "generalized harmonic spectral analysis" [9], which is based on the calculation of an autocorrelation function, and the "chi-square periodogram" [10], which is a modified version of Enright's periodogram.

The locomotor activity patterns of a typical male and a typical female rat of the inbred strains LEW/Ztm and $\mathrm{BH} / \mathrm{Ztm}$ are compared in Fig. 1. Al- 
though all animals were nocturnal, the most obvious differences between strains and sexes were apparent during the dark period. The LEW and $\mathrm{BH}$ females exhibited a more or less constant level of activity during the dark period, while the males were characterized by a trimodal (LEW) or bimodal $(\mathrm{BH})$ activity pattern. These activity distributions give rise to ultradian frequencies of 4 and $4.8 \mathrm{~h}$ period length for the LEW male and 4, 4.8, and $6 \mathrm{~h}$ period length for the $\mathrm{BH}$ male. In the activity patterns of the BH and LEW females only a weak $12-\mathrm{h}$ rhythm, but no ultradian periodicities were detected by either data analysis method.

This sex difference applies equally to all animals of the particular strain. As the harmonic spectral analysis allows pooling of spectral estimates of several
$\mathrm{BH}$ वे LD
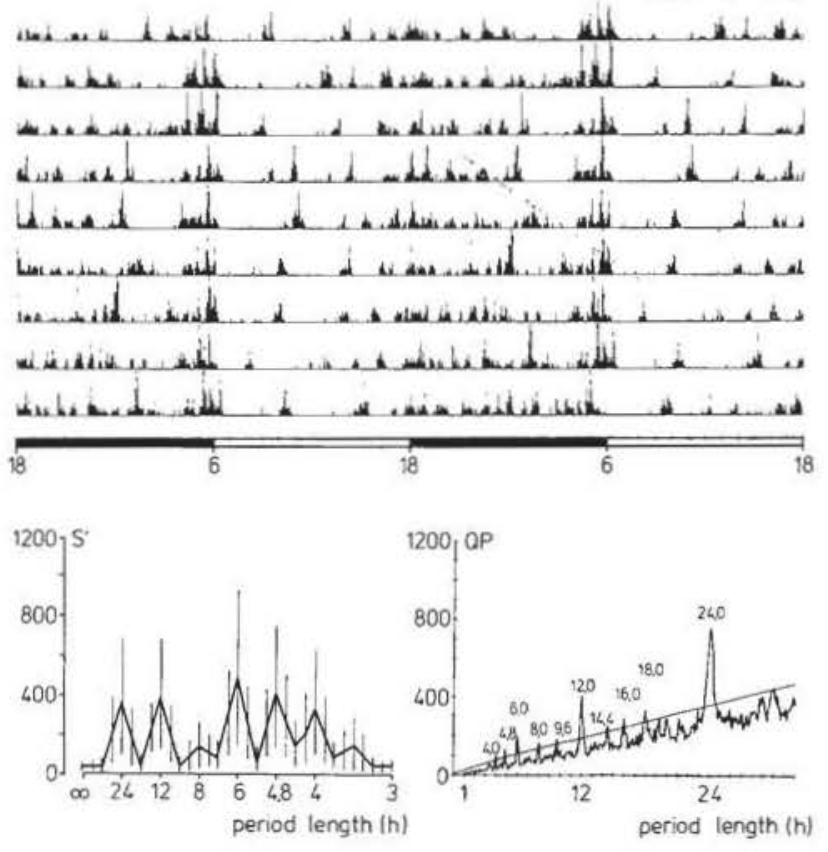

LEW $\sigma^{\circ} \quad$ LD

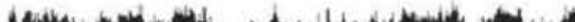

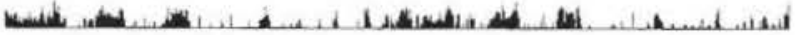

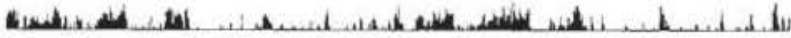

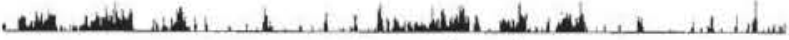

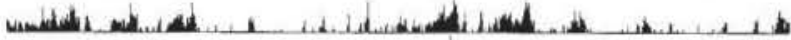

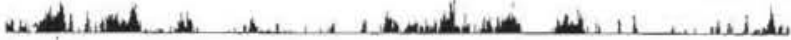

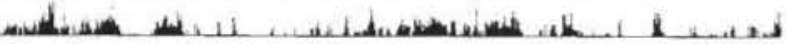

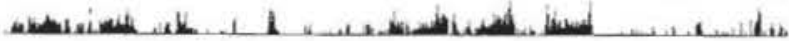

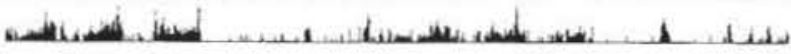
\begin{tabular}{lllll}
\hline 18 & 6 & 18 & 6
\end{tabular}
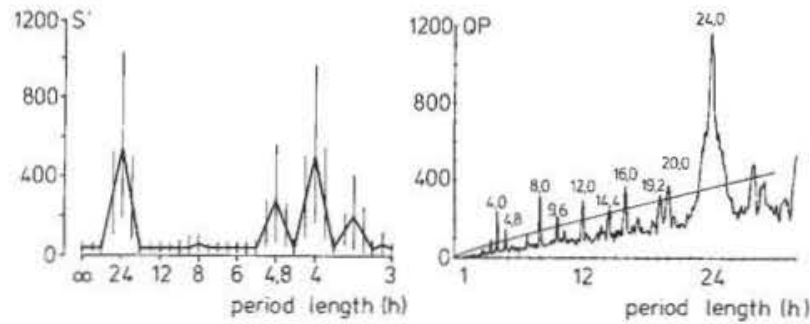

$\mathrm{BH} \& \mathrm{LD}$
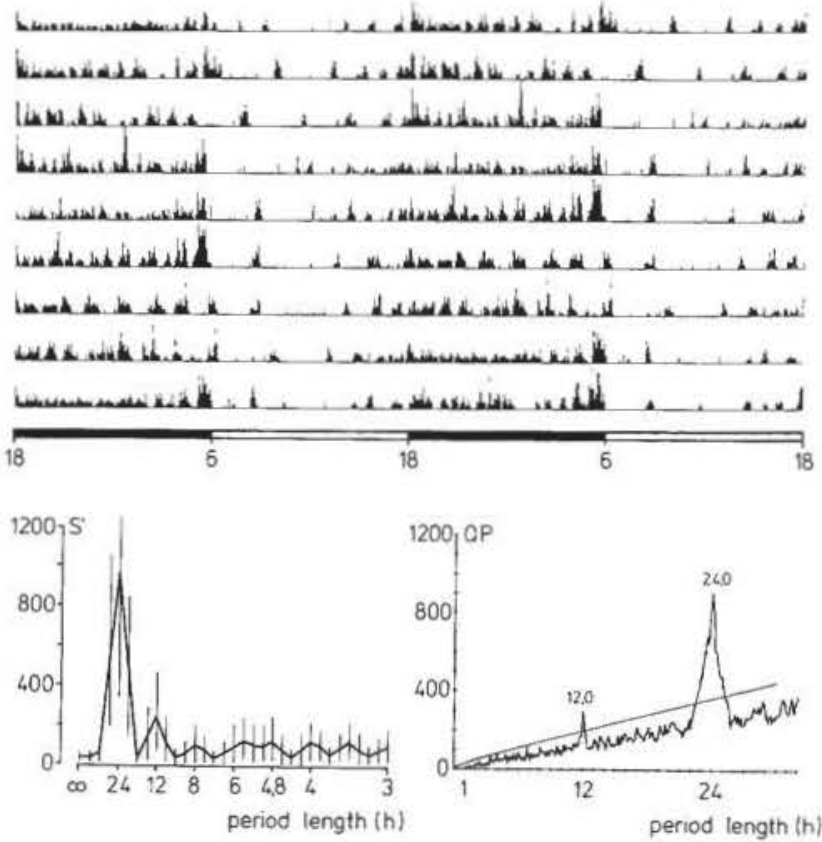

LEW \& LD
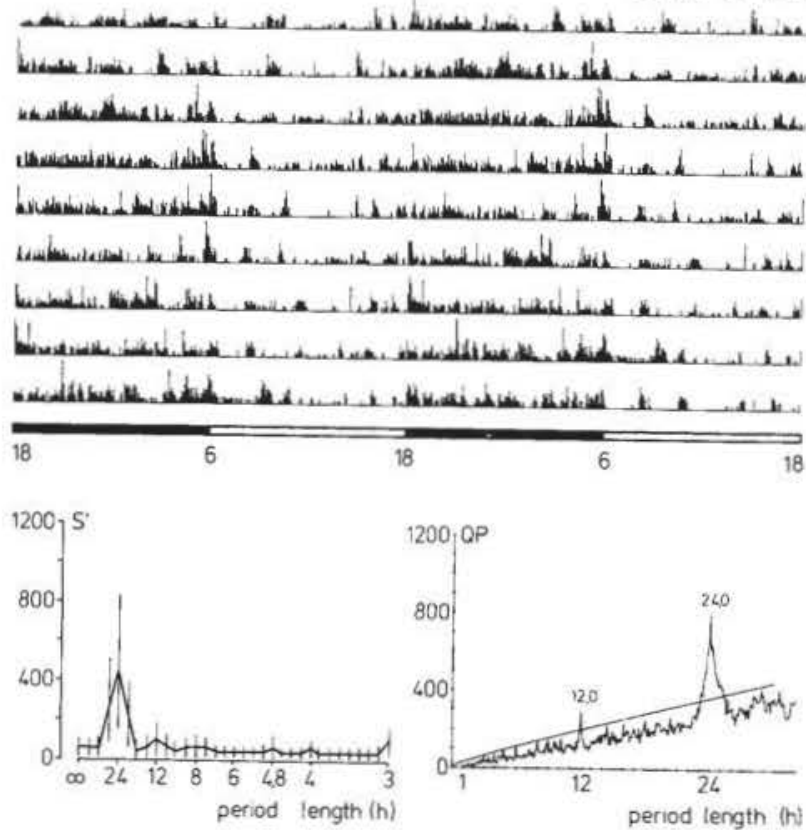

Fig. 1. Double-plotted locomotor activity records of a typical male and a typical female rat of the inbred strains BH/Ztm and LEW/Ztm entrained to LD 12:12. Below the records of each animal the spectral analysis of these individual data is shown on the left, the chi-square periodogram on the right side 

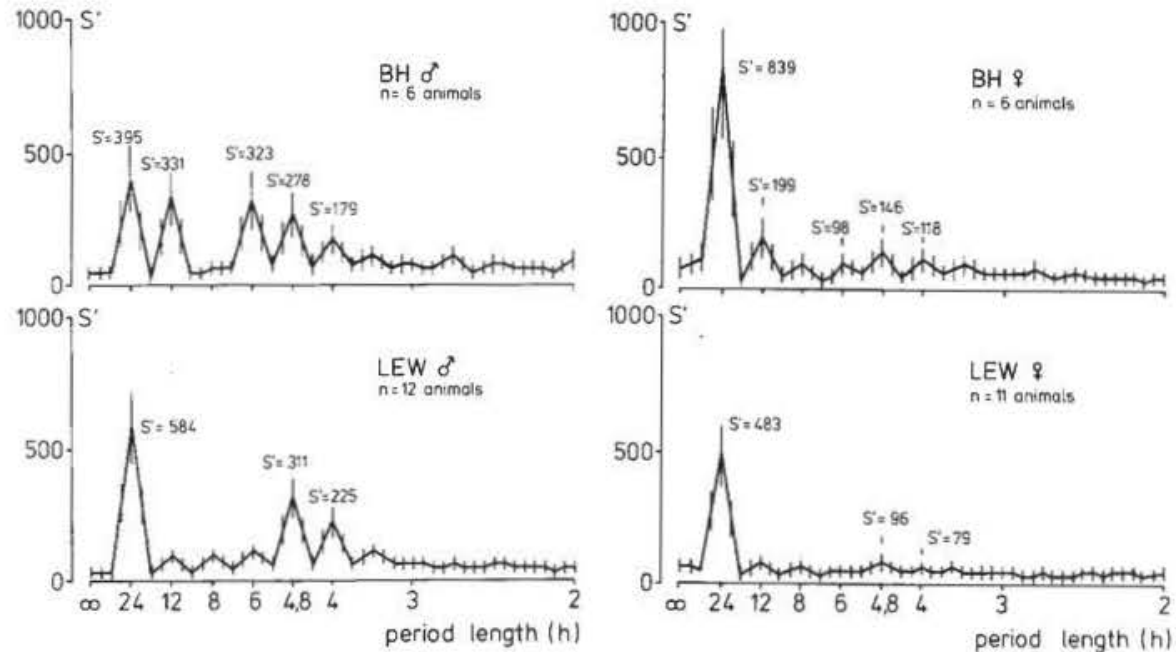

Fig. 2. Pooled spectral analysis of locomotor activity within both sexes of the inbred strains $\mathrm{BH} / \mathrm{Ztm}$, and LEW $/ \mathrm{Ztm}$. The $95 \%$ confidence limits of the spectral estimates of $n$ animals are plotted as vertical lines

individuals, animals of the same strain and sex can be grouped together and the groups compared with each other. The pooled spectral analysis of both male and female rats of the two inbred strains are shown in Fig. 2. Differences in the amplitude of distinct spectral estimates were examined with Student's $t$-test.

The $\mathrm{BH}$ strain showed significant sex differences in regard to the $24-\mathrm{h} \mathrm{com}$ ponent, the 24-h component of the females $\left(S^{\prime}=839 \pm 212\right)$ being twice that of the males $\left(S^{\prime}=395 \pm 235\right)$. Moreover, the spectral analysis of the male $\mathrm{BH}$ rats included ultradian components of $12 \mathrm{~h} \quad\left(S^{\prime}=331 \pm 144\right), 6 \mathrm{~h}$ $\left(S^{\prime}=323 \pm 271\right), 4.8$ h $\left(S^{\prime}=278 \pm 199\right)$, and $4 \mathrm{~h}\left(S^{\prime}=179 \pm 90\right)$ period lengths. The amplitudes of these periods were significantly higher than corresponding amplitudes in the spectrum of the $\mathrm{BH}$ females.

The spectral analysis of the LEW strain showed a 24-h component which did not differ significantly between males $\left(S^{\prime}=584 \pm 132\right)$ and females $\left(S^{\prime}=\right.$ $483 \pm 164)$. Additional ultradian components of $4.8\left(S^{\prime}=311 \pm 92\right)$ and $4 \mathrm{~h}$ $\left(S^{\prime}=225 \pm 135\right)$ period lengths occurred only in male animals.

Sex differences in the daily pattern of locomotor activity were observed in two inbred strains of laboratory rats. The amplitude of the 24-h rhythm was significantly lower in male rats of the $\mathrm{BH}$ strain than in females of this strain. Although this observation is limited to only one strain, it supports earlier reports which suggested sex-related differences in the circadian system of the rat $[1,3,4]$. Female rats have been found to show daily rhythmicity in neuroendocrine functions, related to the timing of ovulation, not seen in males [3]. Females, likewise, have a higher plasma corticosterone concentration peak than males [4].

Since ultradian rhythms in locomotor activity were observed only in male animals of the LEW and BH strains, the data additionally indicate that ultradian rhythms in locomotor activity are sex-specific. Ultradian rhythms are known to exist in laboratory rats [11, 12], and it has been shown that their existence is connected with a distinct genetical background [7]. Causal and functional analyses of ultradian rhythms, however, have only just begun.

Measurements performed on rats of the BH and LEW strains under continuous light conditions confirmed the assumption of an independent ultradian oscillator which causes activity rhythms in the LEW and BH strains [7]. Other authors [13], however, suggest that ultradian rhythmicity reflects the activity of several circadian oscillators coupled to one another in various phase relations (i.e. $4.8 \mathrm{~h}$ or $4 \mathrm{~h}$ apart). The known multioscillator structure of the pacemaker system provides several possibilities concerning the specific nature of the observed ultradian rhythms, which might also result from the different hormonal environments of the sexes.

Testosteron, for instance, plays an important role in the circadian organization. This is suggested by the observation that in starlings testosterone provoked "splitting" of the activity rhythms into two components, which temporarily free-ran with different circadian frequencies [14]. Testosterone may, therefore, be involved in the coupling of circadian oscillators controlling the rhythm of locomotor activity. A recent report on female hamsters also indicated that gonadal steroids may play a role in synchronizing a multioscillatory circadian system. In this particular study, however, splitting was associated with low estrogen levels [15]. As is the case with many other sexually differentiated behaviors [16], the hormonal environment during a critical stage of development determines how the adult circadian system will respond to hormonal signals [5, 17].

Further studies are needed to understand the nature of ultradian rhythms and to determine the extent to which the difference between male and female rats may depend on the adult or perinatal hormonal environment.

I thank S. Lühmann for her help and technical assistance, and K.D. Döhler, K. Gärtner, T.R. Geimer and A. Herrmann for their comments on the manuscript. This research was supported by a grant from the Deutsche Forschungsgemeinschaft, SFB 146.

Received October 31 and December 10, 1984

1. Turek, F.W., Gwinner, E., in: Vertebrate Circadian Systems, p. 173 (eds. Aschoff, J., Daan, S., Groos, G.A.). Berlin-Heidelberg: Springer 1982

2. Gorski, R.A., et al.: Brain Res. 148, 333 (1978)

3. Brown-Grant, K.: J. Endocrinol. 62, 319 (1974)

4. Critchlow, V., et al.: Am. J. Physiol. 205, 807 (1963)

5. Zucker, I., et al.: ibid. 238, R97 (1980)

6. Davis, F.D., et al.: ibid. 244, R93 (1983)

7. Büttner, D., Wollnik, F.: Behav. Genet. 14 (2), 137 (1984)

8. Plonait, H., et al.: Z. Versuchstierk. 24, 244 (1982)

9. Halberg, F., Panofsky, H.: Exp. Med. Surg. 19, 285, 323 (1961)

10. Sokolove, P.G., Bushell, W.N.: J. Theor. Biol. 72, 131 (1978) 
11. Honma, K., Hiroshige, T.: Jap. J. Physiol. 28, 159 (1978); Am. J. Physiol. 235, R243 (1978)

12. Stephan, F.K.: Physiol. Behav. 30, 451 (1983)

13. Rusak, B.: Fed. Proc. 38, 2589 (1979)

14. Gwinner, E.: Science 185,72 (1974)

15. Morin, L.P.: Physiol. Behav. 24, 741 (1980)
16. Goy, R.W., Goldfoot, D.A., in: Handbook of Physiology, Endocrinology, p. 169. Washington DC: Am. Physiol. Soc. 1973

17. Zucker, L, in: Biological Rhythms and their Central Mechanism, p. 369 (eds. Suda, M., Hayaishi, O., Nakagawa, N.). Amsterdam: Elsevier/North-Holland, Biomed. Press 1979 